\title{
Synthesis of Defined Ubiquitin Dimers
}

\author{
Silvia Eger, Martin Scheffner, ${ }^{*}$ Andreas Marx, ${ }^{*}$ and Marina Rubini ${ }^{*}$ \\ Department of Chemistry, Department of Biology, and Konstanz Research School Chemical Biology, University of \\ Konstanz, 78457 Konstanz, Germany
}

Received August 13, 2010; E-mail: martin.scheffner@uni-konstanz.de; andreas.marx@uni-konstanz.de; marina.rubini@uni-konstanz.de

\begin{abstract}
Many proteins are post-translationally modified by the attachment of poly-ubiquitin (Ub) chains. Notably, the biological function of the attached Ub chain depends on the specific lysine residue used for conjugate formation. Here, we report an easy and efficient method to synthesize site-specifically linked Ub dimers by click reaction between two artificial amino acids. In fact, we were able to synthesize all seven naturally occurring Ub connectivities, providing the first example of a method that gives access to all Ub dimers. Furthermore, these synthetic Ub dimers are recognized by the natural ubiquitination machinery and are proteolytically stable, making them optimal candidates to further investigate the function of differently linked Ub chains.
\end{abstract}

Ubiquitin (Ub) first attracted great attention when the Ubmediated protein degradation pathway was discovered in the late 1970s. ${ }^{1}$ Today, we know that in addition to its function in protein degradation, Ub also has non-proteolytic functions and plays a fundamental role in the control of many biological processes, including cell cycle regulation, transcription, DNA repair, and apoptosis. $^{2}$

Substrate proteins are often modified by the attachment of polyUb chains. Poly-Ub chains consist of several Ub moieties that are linked to each other via isopeptide bonds between a specific lysine residue of one $\mathrm{Ub}$ and the $\mathrm{C}$-terminal glycine of the next $\mathrm{Ub}$. $\mathrm{Ub}$ contains seven lysine residues (Lys6, Lys11, Lys27, Lys29, Lys33, Lys48, and Lys63), each of which can be used for poly-Ub chain formation. ${ }^{3}$ It is important to note that the actual lysine residue of $\mathrm{Ub}$ used for $\mathrm{Ub}-\mathrm{Ub}$ conjugation is assumed to determine the biological function of the respective poly-Ub chain. ${ }^{4}$

Today, it is possible to enzymatically synthesize Lys48- and Lys63-linked poly-Ub chains ${ }^{5}$ that can be employed in structural and functional studies. However, they are less suited for experiments with whole-cell lysates, since these contain Ub-specific proteases that efficiently cleave natural Ub conjugates. ${ }^{6}$ Furthermore, this approach is limited by low productivity and by the availability of respective enzymes. Recently, several methods for site-specific $\mathrm{Ub}$ protein conjugation were reported, including oxime-based ligation $^{7}$ and native chemical ligation. ${ }^{8}$ The $\mathrm{Cu}(\mathrm{I})$-catalyzed Huisgen azide-alkyne cycloaddition (click reaction) ${ }^{9}$ was used to link Ub and several Ub-like proteins (Ubl's) to a model protein. ${ }^{10} \mathrm{~A}$ thioether ligation approach was explored for the synthesis of a Ub dimer. $^{11}$

Here we report on the synthesis of all seven possible ubiquitin dimers by linking the $\mathrm{C}$-terminus of one $\mathrm{Ub}$ to the respective natural linkage position of a second Ub via click reaction. Our approach is based on incorporating an azide and an alkyne, respectively, into $\mathrm{Ub}$ via artificial amino acids and linking them by click reaction (Figure 1). The azide function was introduced via the methionine analogue azidohomoalanine (Aha) ${ }^{12}$ (Figure 2a) and the alkyne function via a pyrrolysine analogue, the propargyl-protected lysine derivative $\mathrm{Plk}^{7,13}$ (Figure $3 \mathrm{a}$ ). The click reaction between the two entities results in a triazole linkage of both molecules. Of note, it is unlikely that replacement of the natural isopeptide bond by a triazole linkage disturbs the natural $\mathrm{Ub}-\mathrm{Ub}$ conformation, since it has been reported that the isopeptide linkage region is generally disordered. ${ }^{14}$

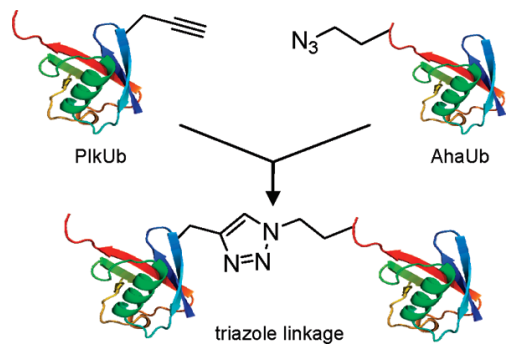

Figure 1. Schematic depiction of Ub dimer formation by Click reaction between an azide-functionalized $\mathrm{Ub}(\mathrm{AhaUb})$ and an alkyne-functionalized $\mathrm{Ub}(\mathrm{PlkUb})$.

First, we introduced Aha at the C-terminus of Ub by substituting the codon for the C-terminal Gly by a Met codon (Figure $2 \mathrm{a}-\mathrm{c}$ ). At the same time, we wanted to eliminate the N-terminal Met, as a second incorporated azide would foil the envisaged site-specific approach. It is known that, in more than half of the Escherichia coli proteins, the N-terminal Met is co-translationally removed by methionine aminopeptidase (MetAP). However, activity of MetAP is dependent on the second and third amino acids of the protein. Small amino acids like Ala, Gly, and Ser facilitate N-terminal Met excision (NME), while bulky amino acids inhibit the process. ${ }^{15}$ It was shown that this rule also applies to the non-natural amino acid Aha. ${ }^{16}$ In Ub, the second and third amino acids are Gln and Ile, respectively, and thus, the initial Met is retained upon translation. In order to achieve removal of the first amino acid, we introduced Ala and Ser between the initial Met and Gln at position 2. This construct (MASUb76M, Figure 2b) was expressed in Met auxotrophic E. coli B834(DE3). Cells were cultured under limiting Met concentration until they reached stationary growth. The growth medium was then switched to fresh minimal medium containing Aha, and protein expression was induced. Indeed, we were able to isolate the target protein with the initial amino acid quantitatively cleaved off and the C-terminal Met completely replaced by Aha, as confirmed by ESI-MS (Figure 2d,e).

Having a Ub derivative with a selectively introduced azide at a single position in hand, we next tested its potential for click reactions and employed an alkyne-functionalized fluorophore and an alkyne-functionalized biotin. Both were successfully used to label the Ub sample (see Supporting Information, Figure S1). These 
a)<smiles>N#CCC(N)C(=O)O</smiles>

b) 1 MASQIFVKILIG KTITLEVEPS 21 DTIENUKAKI QDKEGIPPDQ 41 QRLIFAGKQL EDGRTLSDYM 61 IQKESTLHLV LRLRGM

azidohomoalanine (Aha)

d)

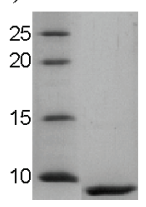

e) ESI-MS analysis of $76 \mathrm{AhaUb}$ (calc: 8660.9 )
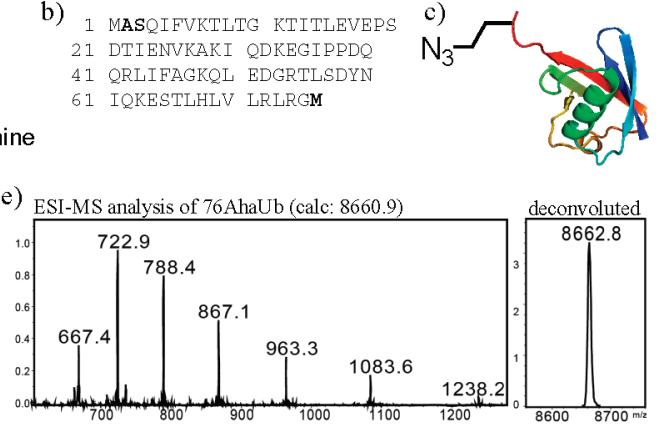

Figure 2. Expression of 76AhaUb: (a) azidohomoalanine (Aha), (b) amino acid sequence of MASUb76MUb, (c) scheme of 76AhaUb, (d) SDS-PAGE gel of 76AhaUb, and (e) ESI-MS and deconvoluted MS spectra of 76AhaUb.

experiments demonstrated that the azide is situated on the surface of the protein and that it is accessible for chemical reactions. Of note, in contrast to commercially available, unspecifically biotinylated $\mathrm{Ub}$, this procedure allows us to label Ub site-selectively with biotin (other positions were also investigated, data not shown). Furthermore, we used these systems to optimize the click reaction conditions. Copper sulfate in the presence of tris(2-carboxyethyl)phosphine (TCEP) as a reducing agent and tris[(1-benzyl-1,2,3triazol-4-yl)methyl]amine (TBTA) as a $\mathrm{Cu}(\mathrm{I})$-stabilizing agent worked best. ${ }^{10}$

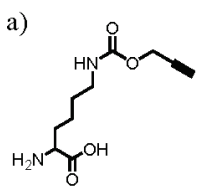

b) 1 MOIFVKTLTG KTITLEVEPS

21 DTIENVKAKI QDKEGIPPDO

41 ORLIFAGROL EDGRTLSDYN

61 IQKESTLHLV LRLRGG

$K$ AAA $\rightarrow$ TAG

pyrrolysine analog

(Pk)

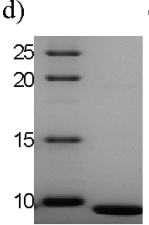

e) ESI-MS analysis of 11 PlkUb (calc: 8646.8 )
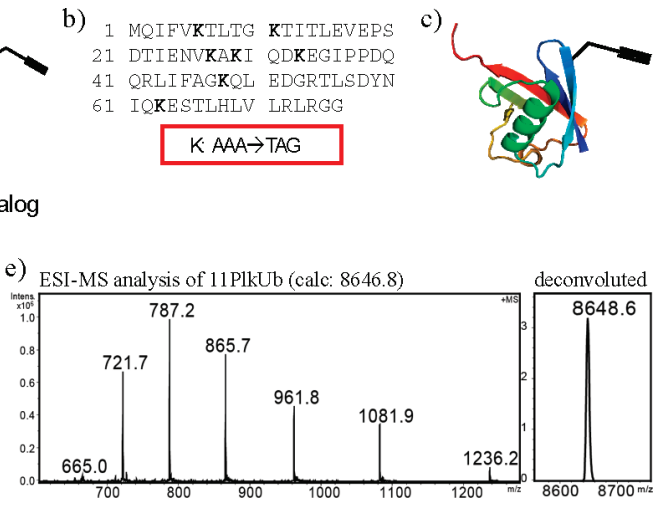

Figure 3. Expression of 11PlkUb: (a) pyrrolysine analogue (Plk), (b) mutation scheme for PlkUb, (c) scheme of 11PlkUb, (d) SDS-PAGE gel of $11 \mathrm{PlkUb}$, and (e) ESI-MS and deconvoluted MS spectra of $11 \mathrm{PlkUb}$.

The second aim was to incorporate an alkyne functionality via the pyrrolysine analogue Plk at each of the seven lysine positions (Figure 3a,b). Pyrrolysine analogues can be incorporated through the amber stop codon UAG via a pyrrolysyl-tRNA synthetase/ tRNA $_{\text {CUA }}$ pair from Methanosarcina barkeri in E. coli. ${ }^{7,13}$ Each of the seven lysine codons of wtUb was separately mutated to the amber stop codon TAG. The gene for the pyrrolysine tRNA synthetase pylS was obtained from genomic DNA from $M$. barkeri and cloned into pRSFduet. The gene for the pyrrolysine tRNA was synthesized together with the lipoprotein promoter lpp and cloned into the backbone of pET11a already containing the gene for $\mathrm{Ub}$ (TAG-Ub) in its multiple cloning site. Both vectors were cotransformed into BL21(DE3) for expression. By this method, we were able to incorporate Plk at all seven lysine positions (11Plk$\mathrm{Ub}$ is shown in Figure 3d,e as an example).

To show that the modified Ub's are still biochemically active, we performed an auto-ubiquitination assay with E6-AP (see Supporting Information, Figure S2). ${ }^{17}$ This process relies on the presence of functional $\mathrm{Ub}$ and can be visualized on SDS-PAGE gels where high-molecular-mass bands representing ubiquitinated forms of E6-AP arise, whereas the intensity of the band at $95 \mathrm{kDa}$ representing the non-modified form E6-AP diminishes. As expected, only Ub derivatives with an internal artificial amino acid were active in this assay, but not the samples in which the C-terminus is modified. When a Lys position is modified, the respective Ub can still be activated and attached to proteins by isopeptide linkage formation. Furthermore, poly-Ub chains can still be formed by using the six remaining Lys residues. However, when the C-terminus of $\mathrm{Ub}$ is modified, it can no longer be activated by E1 and, thus, cannot be attached to other proteins.

By the above approaches, we were able to express and purify an azide-functionalized $\mathrm{Ub}$ (76AhaUb) and seven alkyne-functionalized Ub's (6PlkUb, 11PlkUb, 27PlkUb, 29PlkUb, 33PlkUb, $48 \mathrm{PlkUb}$, and 63PlkUb) in large quantities. Click reactions were set up with a 1:1 ratio of 76AhaUb and each of the seven PlkUb's. Analysis of the reactions by SDS-PAGE showed the formation of a new band at about 13-14 kDa (Figure 4a) that was not detectable in the negative control experiment, where only one of the two Ub species was present (data not shown). As anticipated, this newly formed band stained positive in a Western blot with anti-Ub antibodies (data not shown). Trypsin digest of the dimer bands followed by MS analysis confirmed the presence of all seven triazole-linked peptide fragments (11PlkUb-76AhaUb is shown as example in Figure 4b; other combinations are shown in Supporting Information, Figures S3-S8). The respective Ub dimers were purified from the two reactants by size exclusion chromatography and analyzed by ESI-MS (Figure 4c,d, 11PlkUb-76AhaUb).

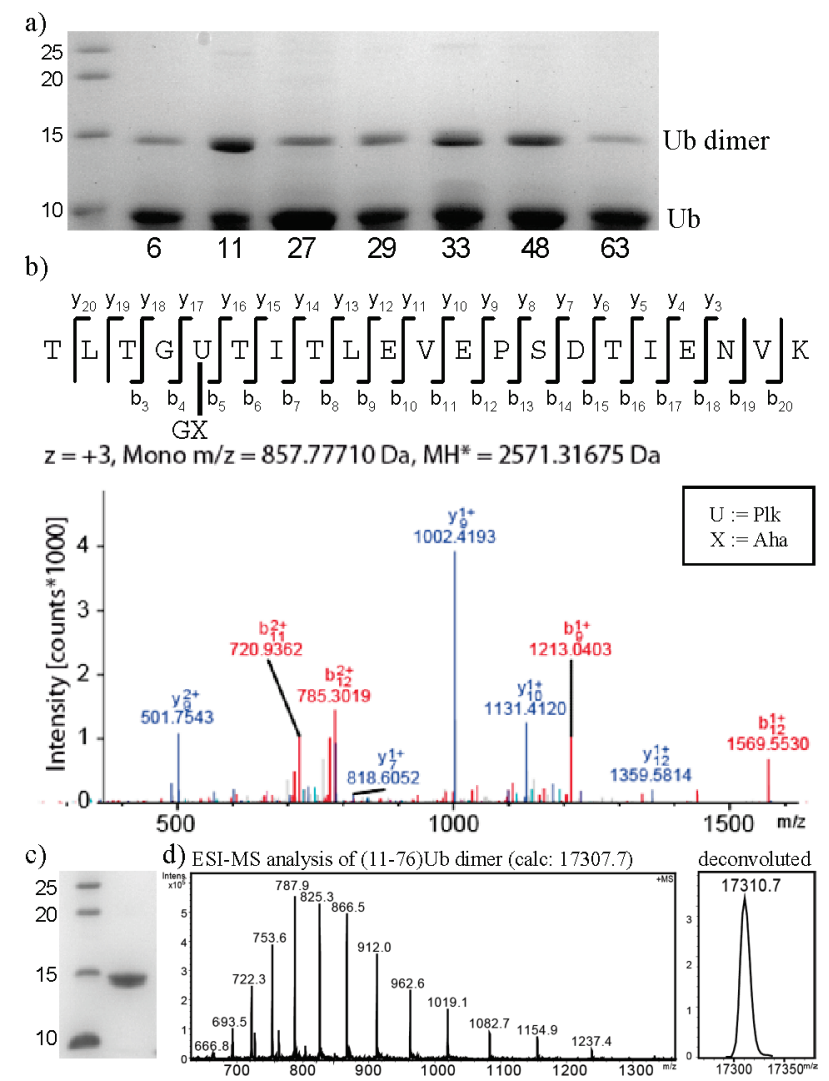

Figure 4. Ub dimer by click reaction: (a) SDS-PAGE gel of click reaction between 76AhaUb and 6PlkUb, 11PlkUb, 27PlkUb, 29PlkUb, 33PlkUb, 48PlkUb, and 63PlkUb; (b) MS/MS spectrum of trypsin-digested 11PlkUb76AhaUb [all b- and $\mathrm{y}$-ions marked in the peptide sequence were found in the corresponding MS/MS spectrum]; (c) SDS-PAGE gel of purified Ub dimer 11PlkUb-76AhaUb; and (d) ESI-MS and deconvoluted MS spectra of $11 \mathrm{PlkUb}-76 \mathrm{AhaUb}$. 


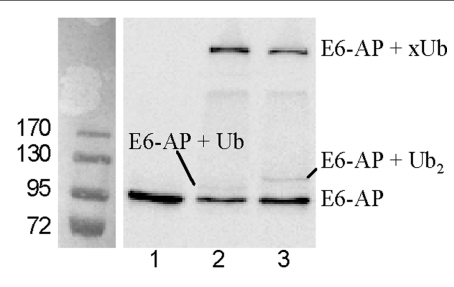

Figure 5. Activity testing of synthetic Ub dimer (11PlkUb-76AhaUb) via E6-AP auto-ubiquitination assay: lane 1, without Ub; lane 2, with wtUb, $(+x U b)$; and lane 3, with Ub dimer (11PlkUb-76AhaUb). Running positions of non-modified E6-AP (E6-AP), mono-ubiquitinated E6-AP (+Ub), E6AP modified by one Ub dimer $\left(+\mathrm{Ub}_{2}\right)$, and poly-ubiquitinated E6-AP $(+x U b)$ are indicated.

To determine if the synthetic Ub dimer is still biochemically active, we performed E6-AP auto-ubiquitination assays with the dimer as the only source of $\mathrm{Ub}$. The appearance of the highmolecular-mass band in lane 3 of Figure 5 shows that the synthetic $\mathrm{Ub}$ dimer can still be used for poly-Ub chain formation, indicating that the synthetic Ub dimer is still recognized by the natural ubiquitination machinery (i.e., the unmodified C-terminus of $11 \mathrm{PlkUb}$ and the unmodified lysine positions can still be used for ubiquitin activation and subsequent conjugation reactions, respectively).

In summary, we established an easy and efficient method to form site-specifically linked Ub dimers that are still recognized by ubiquitinating enzymes. To our knowledge, this is the first description of a method that gives access to all seven possible linkages, laying the foundation for further investigations of the function of differently linked Ub chains. In the future, these Ub dimers can, for example, be used to generate linkage specific antibodies or to identify new linkage-specific interaction partners. Furthermore, this method is not restricted to $\mathrm{Ub}$ and allows linking any two proteins in a site-specific manner.

Acknowledgment. We gratefully acknowledge funding by the Konstanz Research School Chemical Biology, DFG, EU-network of excellence RUBICON. S.E. is grateful for a grant by the Boehringer Ingelheim Fonds. We thank A. Marquardt for expert assistance with mass spectrometry and T. Kapitza with E6-AP assays.

Supporting Information Available: Click reaction of Ub with biotin and fluorescent dye; E6-AP auto-ubiquitination assay with Ub mono- mers; MS/MS spectra of tryptic fragments of synthetic Ub dimers; detailed experimental procedures. This material is available free of charge via the Internet at http://pubs.acs.org.

\section{References}

(1) Hershko, A.; Ciechanover, A. Annu. Rev. Biochem. 1992, 61, 761-807.

(2) (a) Hershko, A.; Ciechanover, A. Аnnu. Rev. Biochem. 1998, 67, 425479. (b) Hicke, L.; Dunn, R. Annu. Rev. Cell. Dev. Biol. 2003, 19, 141172. (c) Pickart, C. M. Annu. Rev. Biochem. 2001, 70, 503-533. (d) Kerscher, O.; Felberbaum, R.; Hochstrasser, M. Annu. Rev. Cell Dev. Biol. 2006, 22, 159-180.

(3) Peng, J.; Schwartz, D.; Elias, J. E.; Thoreen, C. C.; Cheng, D.; Marsischky, G.; Roelofs, J.; Finley, D.; Gygi, S. P. Nat. Biotechnol. 2003, 21, $921-$ 926.

(4) Li, W.; Ye, Y. Cell Mol. Life Sci. 2008, 65, 2397-2406.

(5) (a) Hofmann, R. M.; Pickart, C. M. J. Biol. Chem. 2001, 276, 2793627943. (b) Pickart, C. M.; Raasi, S. Methods Enzymol. 2005, 399, 21-36.

(6) Nijman, S. M. B.; Luna-Vargas, M. P. A.; Velds, A.; Brummelkamp, T. R.; Dirac, A. M. G.; Sixma, T. K.; Bernards, R. Cell 2005, 123, 773-786.

(7) Shanmugham, A.; Fish, A.; Luna-Vargas, M. P.; Faesen, A. C.; El Oualid, F.; Sixma, T. K.; Ovaa, H. J. Am. Chem. Soc. 2010, 132, 8834-8835.

(8) (a) McGinty, R. K.; Kim, J.; Chatterjee, C.; Roeder, R. G.; Muir, T. W. Nature 2008, 453, 812-6. (b) Li, X.; Fekner, T.; Ottesen, J. J.; Chan, M. K. Angew. Chem., Int. Ed. 2009, 48, 1-5. (c) Kumar, K. S. A.; Haj-Yahya, M.; Olschewski, D.; Lashuel, H. A.; Brik, A. Angew. Chem., Int. Ed. 2009, 48, 8090-8094. (d) Erlich, L. A.; Kumar, K. S. A.; Haj-Yahya, M.; Dawson, P. E.; Brik, A. Org. Biomol. Chem. 2010, 8, 2392-2396. (e) Kumar, K. S. A.; Spasser, L.; Erlich, L. A.; Bavikar, S. N.; Brik, A.; Angew. Chem., Int. Ed. DOI: $10.1002 /$ ange. 201003763

(9) (a) Huisgen, R. Angew. Chem., Int. Ed. Engl. 1963, 2, 565-598. (b) Rostovtsev, V. V.; Green, L. G.; Fokin, V. V.; Sharpless, K. B. Angew. Chem., Int. Ed. 2002, 41, 2596-2599. (c) Tornoe, C. W.; Christensen, C.; Meldal, M. J. Org. Chem. 2002, 67, 3057-3064.

(10) Weikart, N.; Mootz, H. ChemBioChem 2010, 11, 774-777.

(11) Jung, J. E.; Wollscheid, H.-P.; Marquardt, A.; Manea, M.; Scheffner, M.; Przybylski, M. Bioconjugate Chem. 2009, 20, 1152-1162.

(12) (a) Kiick, K. L.; Saxon, E.; Tirrell, D. A.; Bertozzi, C. R. Proc. Natl. Acad. Sci. U.S.A. 2002, 99, 19-24. (b) Kasteren, S. I. v.; Kramer, H. B.; Jensen, H. H.; Campbell, S. J.; Kirkpatrick, J.; Oldham, N. J.; Anthony, D. C.; Davis, B. G. Nature 2007, 446, 1105-1109.

(13) (a) Kaya, E.; Gutsmiedl, K.; Vrabel, M.; Müller, M.; Thumbs, P.; Carell, T. ChemBioChem 2009, 10, 2858-2861. (b) Nguyen, D. P.; Lusic, H.; Neumann, H.; Kapadnis, P. B.; Deiters, A.; Chin, J. W. J. Am. Chem. Soc. 2009, 131, 8720-8721. (c) Fekner, T.; Li, X.; Lee, M. M.; Chan, M. K. Angew. Chem., Int. Ed. 2009, 48, 1633-1635.

(14) Phillips, C. L.; Thrower, J.; Pickart, C. M.; Hill, C. P. Acta Crystallogr. Sect. D 2001, 57, 341-344.

(15) Ben-Bassat, A.; Bauer, K.; Chang, S.-Y.; Myambo, K.; Boosman, A.; Chang, S. J. Bacteriol. 1987, 169, 751-757.

(16) (a) Merkel, L.; Cheburkin, Y.; Wiltschi, B.; Budisa, N. ChemBioChem 2007, 8, 2227-2232. (b) Merkel, L.; Beckmann, H. S. G.; Wittmann, V.; Budisa, D. ChemBioChem 2008, 9, 1220-1224. (c) Wang, A.; Nairn, N. W.; Johnson, R. S.; Tirrell, D. A.; Grabstein, K. ChemBioChem 2008, 9, 324330 .

(17) (a) Nuber, U.; Schwarz, S. E.; Scheffner, M. Eur. J. Biochem. 1998, 254, 643-649. (b) Scheffner, M.; Nuber, U.; Huibregtse, J. M. Nature 1995, $373,81-83$.

JA1072838 